\title{
Giant congenital melanocytic nevus of the upper extremity: Coverage with a pre-expanded pedicled thoracodorsal artery perforator flap and re-expanded transferred flap
}

Hyee Jae Yang, Sang Yoon Kang

Department of Plastic Surgery, Kyung Hee University Hospital, Kyung Hee University College of Medicine, Seoul, Korea
Giant congenital melanocytic nevi are rare, and it is recommended to excise due to the possibility of malignancy. Although tissue expansion for nevus removal involves multiple stages, the advantages of tissue expansion are known well. We report a case of a giant congenital melanocytic nevus of the upper extremity that extended from the elbow to the shoulder. This case was challenging due to the circumferential nature of the lesion, the lack of healthy skin, the need to ensure minimal scarring, and the desire to impact the patient's daily life as little as possible. The use of a pre-expanded thoracodorsal artery perforator (TDAP) flap and re-expansion of the transferred flap were considered most appropriate. A pre-expanded pedicled TDAP flap can be transferred to any anatomical region with its versatility. The re-expansion of the transferred flap ensures the health of the skin and minimizes donor site scarring. Here, we achieved the optimal outcome through the combined use of a pre-expanded pedicled TDAP flap and sequential re-expansion of the transferred flap in the treatment of a giant nevus of the upper extremity.

Keywords Nevus / Pedicled flap / Tissue expansion / Perforator flap

\section{INTRODUCTION}

Giant congenital melanocytic nevi are very rare, with a reported incidence of one in 20,000 births. These lesions are associated with a risk of malignant melanoma and neurocutaneous melanosis and also impact physical appearance. Excision is preferred due to the possibility of malignant melanoma, neurocutaneous melanosis, or aesthetic concerns [1].

Received: Oct 17, 2019 Revised: Nov 10, 2019 Accepted: Nov 11, 2019 Correspondence: Sang Yoon Kang Department of Plastic Surgery, Kyung Hee University Hospital, Kyung Hee University College of Medicine, 23 Kyungheedae-ro, Dongdaemun-gu, Seoul 02447, Korea

Tel: +82-2-958-8431, Fax: +82-2-963-5638, E-mail: nuelk@nate.com

Copyright @ 2020 The Korean Society for Aesthetic Plastic Surgery.

This is an Open Access article distributed under the terms of the Creative Commons Attribution Non-Commercial License (https://creativecommons.org/licenses/by-nc/4.0/) which permits unrestricted non-commercial use, distribution, and reproduction in any medium, provided the original work is properly cited. www.e-aaps.org
Tissue expansion (TE) for the removal of giant congenital melanocytic nevi is performed in multiple stages; nevertheless, the technique is considered a reliable reconstructive method to achieve optimal aesthetic and functional outcomes. TE provides extra skin with a better color and texture match than skin grafts as well as minimal donor site deformity, and it allows for repeated use of the expanding donor site [2]. Reconstruction of the upper extremities can be a major challenge depending on the anatomical site of the lesion, such as the shoulder, axillary, or elbow region, due to the lack of sufficient tissue with adequate elasticity and durability. A thoracodorsal artery perforator (TDAP) flap can be transferred to various anatomical regions because it can be harvested with a long pedicle, is relatively pliable, and protects against continuous mechanical stress. In patients with large lesions, the combination of TE and a TDAP flap provides extra skin to cover the defect and reduces the number of additional procedures required. In cases in which limitations on donor site expansion exist, however, it is necessary to account for the need to perform additional expansion at 
the defect site in order to achieve coverage of the deficit.

We report a case of a giant congenital melanocytic nevus of the left upper extremity, wherein we performed limited expansion of a TDAP flap at the donor site and sequential re-expansion after the pre-expanded pedicled TDAP flap was transferred to the defect that remained after removal of the nevus.

\section{CASE REPORT}

\section{Patient}

In 2017, a 25-year-old woman presented to our clinic with a giant circumferential nevus extending from the elbow joint to the shoulder. The nevus was about $31 \mathrm{~cm}$ long and $22.5 \mathrm{~cm}$ wide, covering $90 \%$ of the entire circumference of the upper arm (Fig. 1). Moreover, the lesion accounted for approximately $5 \%$ of the total body surface area. The giant nevus was present on the left upper extremity at birth, when it measured $21 \times 15 \mathrm{~cm}$, and at the age of 6 years, the patient underwent partial surgical removal of the nevus at the forearm and elbow with a full-thickness skin graft measuring $4.5 \times 19$ $\mathrm{cm}$.

The patient preferred a procedure that would yield optimal results both functionally and aesthetically with minimal disruption to her daily life. We explained that a large flap was required to fully cover the lesion on the upper arm, but the larger the tissue expander, the longer the scar on the donor site, which can be prominent during the expansion period. For these reasons, we scheduled preexpanded pedicled TDAP flap surgery on the patient's left back and staged excision of the lesion through serial re-expansion of the transferred flap on the upper arm. Additionally, we believed that multiple rounds of general endotracheal anesthesia (GEA) would burden the patient; accordingly, we planned to use GEA for the main procedures and conscious sedation for the additional operations.

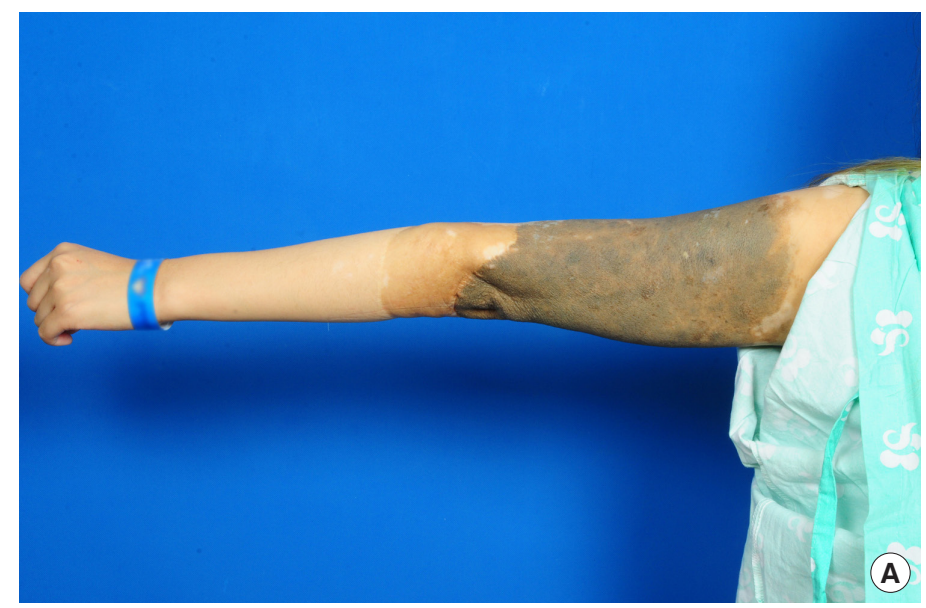

\section{Surgical technique and process}

The operation focused on ensuring free functional movement of the arm, achieving the aesthetic goal of minimal scarring and skin tone matching, and allowing the patient to continue her typical daily activities and wear her usual clothing. Accordingly, we planned to perform sequential TE by combining pre-expansion of a pedicled TDAP flap at the donor site with multiple expansions of the transferred flap on the upper arm. We used a relatively small expander to limit the scarring of the donor site and to allow the pa-

Table 1. Surgical procedures

\begin{tabular}{|c|c|c|c|c|}
\hline $\begin{array}{c}\text { Procedure } \\
\text { number }\end{array}$ & $\begin{array}{l}\text { Type of } \\
\text { anes- } \\
\text { thesia }\end{array}$ & Procedure (mL) & $\begin{array}{l}\text { Nevus } \\
\text { size } \\
\left(\mathrm{cm}^{2}\right)\end{array}$ & $\begin{array}{l}\text { Total volume } \\
\text { of expansion } \\
\text { (inflated/ } \\
\text { original, } \mathrm{mL} \text { ) }\end{array}$ \\
\hline \multirow[t]{2}{*}{ First } & GEA & Expander insertion (760) & $31 \times 22.5$ & $840 / 760$ \\
\hline & & Inflation (120) & & \\
\hline \multirow[t]{5}{*}{ Second } & GEA & Expander removal & $25 \times 12$ & $585 / 560$ \\
\hline & & Expanded flap transfer & & \\
\hline & & Partial resection of nevus & & \\
\hline & & Expander insertion (560) & & \\
\hline & & Inflation (25) & & \\
\hline \multirow[t]{3}{*}{ Third } & Local $^{\text {al }}$ & Partial resection of nevus & $25 \times 5$ & $575 / 560$ \\
\hline & & Expander insertion (560) & & \\
\hline & & Inflation (25) & & \\
\hline \multirow[t]{3}{*}{ Fourth } & Local $^{\text {a) }}$ & Partial resection of nevus & $20 \times 4$ & $580 / 560$ \\
\hline & & Expander insertion (560) & & \\
\hline & & Inflation (30) & & \\
\hline \multirow[t]{2}{*}{ Fifth } & Local $^{\text {a) }}$ & Completion of nevus resection & $20 \times 2$ & \\
\hline & & Expander removal & & \\
\hline
\end{tabular}

GEA, general endotracheal anesthesia.

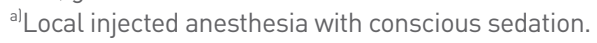

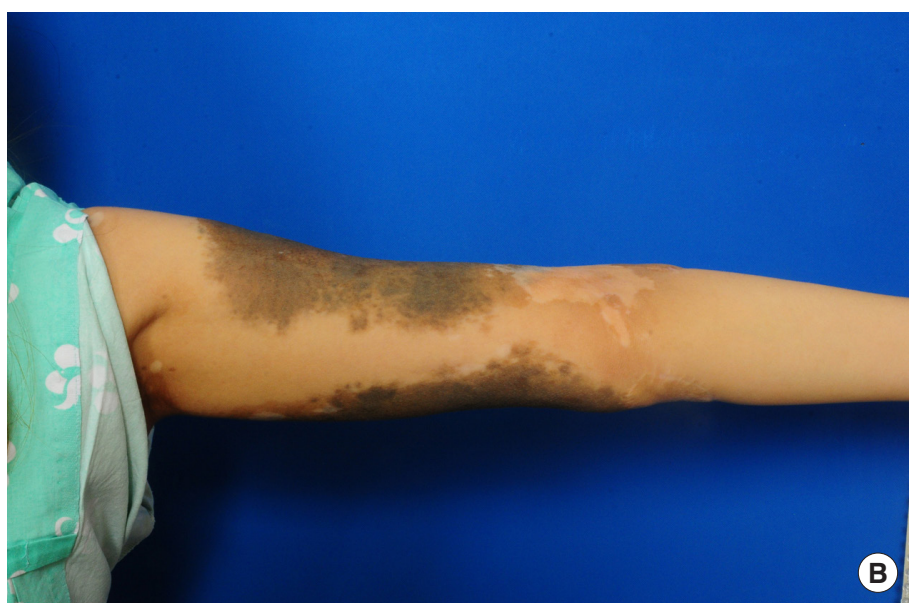

Fig. 1. A 25-year-old woman with a giant circumferential nevus extending from the left elbow joint to the left upper arm. (A) Lateral view. (B) Medial view. 

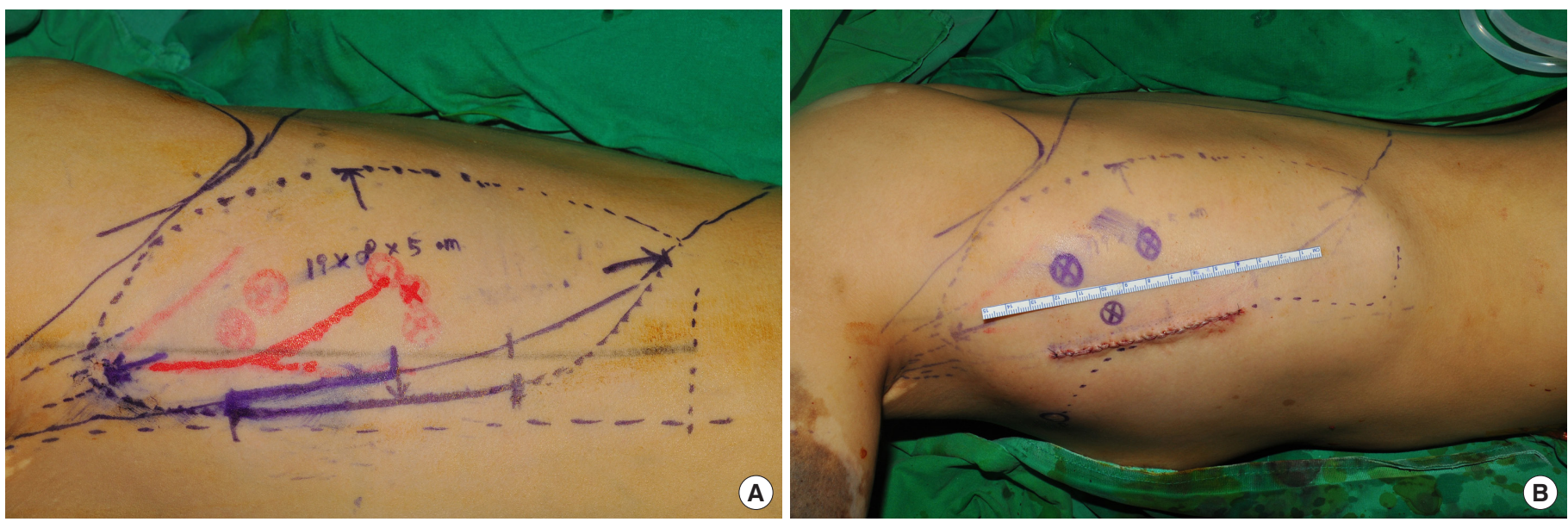

Fig. 2. The first procedure, during which the tissue expander was placed. (A) Preoperative marking. (B) Immediate postoperative view. The 760-mL expander was placed at the suprafascial level.

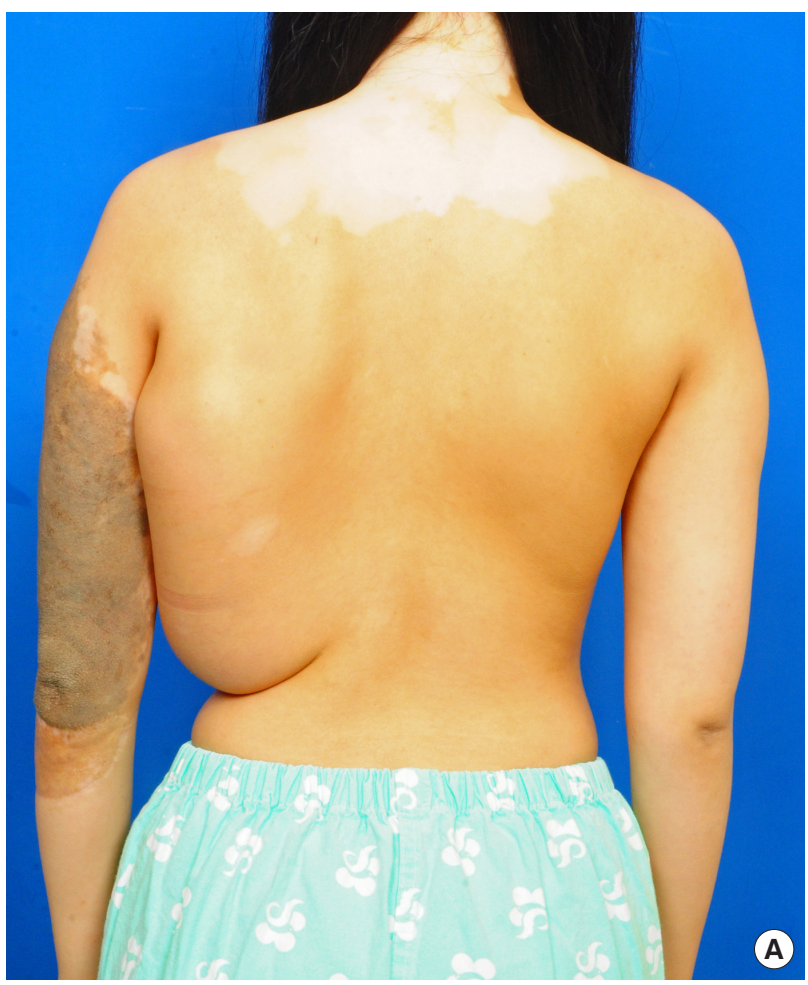

(A)

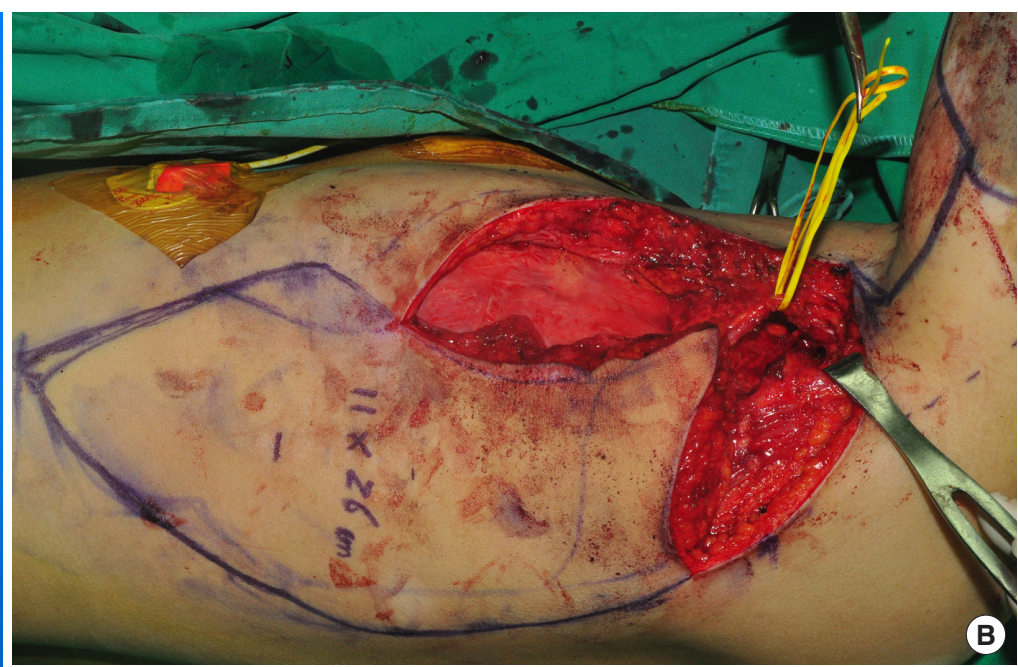

Fig. 3. The second procedure, during which the pre-expanded perforator flap was harvested. (A) Preoperative view of the pre-expanded perforator flap. (B) Intraoperative view of the dissected pedicle.

scending branch via the thoracodorsal artery. The serratus vessels and transverse branches were ligated. After isolation of the pedicle, we created a suprafascial pocket for the tissue expander (rectangular, $19 \times 8 \times 5 \mathrm{~cm}, 760 \mathrm{~mL}$; Sebbin, Boissy-l'Aillerie, France). The bleeding was controlled and the area irrigated, and an expander was placed under the skin flap and perforator pedicle; the expander was then partially inflated (Fig. 2B). Starting 2 weeks after surgery, weekly serial expansions were performed until the expander reached maximal expansion volume. The tissue expander was gradually inflated to a total volume of $840 \mathrm{~mL}$ over 5 weeks. 

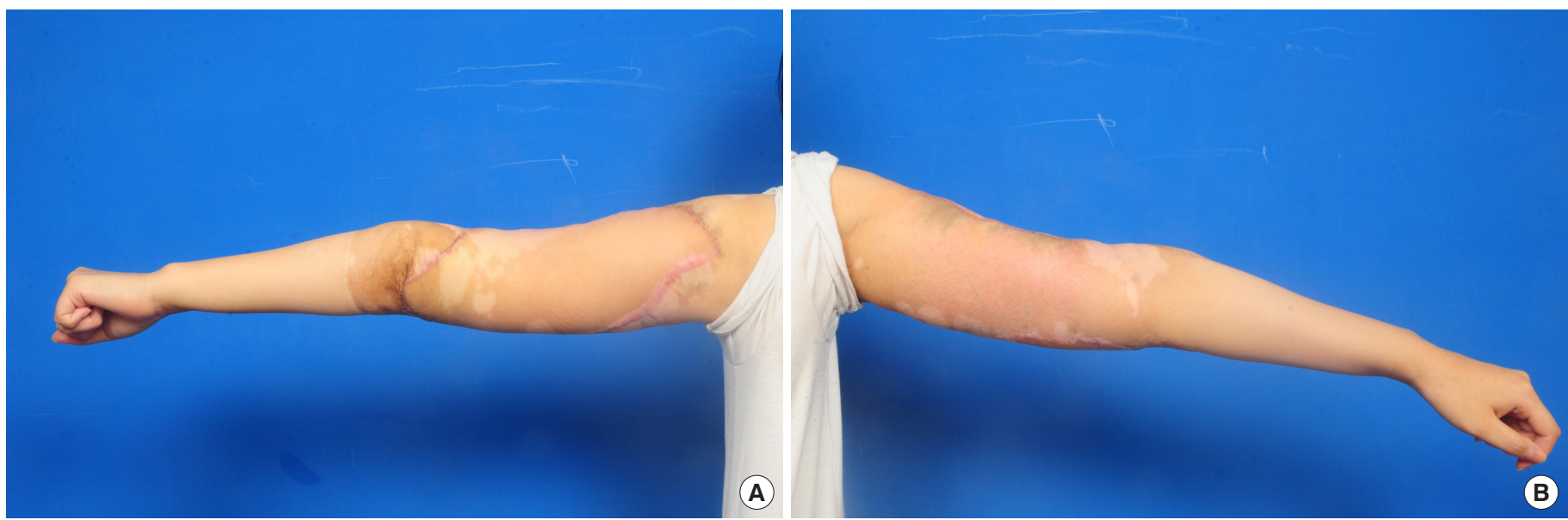

Fig. 4. At 2-month follow-up, postoperative photographs show excellent results. (A) Posterior view. (B) Anterior view.

Twelve weeks after completion of the expansion, a second operation was performed under GEA (Fig. 3). An incision was made along the previous operative scar, and dissection was carried out to expose the tissue expander. With the removal of the expander, we extended the incision and elongated the pedicle until it was long enough for the flap to be transferred without tension or kinking. The muscle around the pedicle was partially included to avoid vessel damage, and the fibrous capsule of the pre-expanded flap was partially excised. After harvest of the pre-expanded pedicled TDAP flap, we excised the nevus at the posterior region of the upper arm and at the elbow, and we covered the defect with the harvested flap. A new tissue expander (rectangular, $16 \times 7 \times 5 \mathrm{~cm}, 560 \mathrm{~mL}$; Sebbin) was placed under the transferred TDAP flap for serial expansion. The donor site was closed primarily without skin tension. Starting 2 weeks after surgery, weekly serial expansions were performed until maximal expansion volume was reached.

Other procedures to remove the rest of the nevus were performed a minimum of 3 weeks after completion of the expansion. This and subsequent procedures (in other words, the third procedure and onward) were performed as day surgery in the outpatient department with the patient under local anesthesia and conscious sedation using propofol (Dongkook Pharm, Seoul, Korea) with secure monitoring of vital signs. After deflation of the expander, the remainder of the nevus was removed with as much flap coverage as possible without tension, and the re-expanded transferred flap was advanced to the area of the defect. During surgery, the expander was removed, and capsulotomy and capsulectomy were performed to allow maximal mobility of the expanded flap. After two additional rounds of serial expansion and excision, the nevus was removed completely. Eventually, through one round of expansion at the donor site and three rounds of transferred flap expansions, we covered the entire lesion of the upper arm with the pre-expanded, serially re-expanded pedicled TDAP flap. The average amount of inflation was $580 \mathrm{~mL}$ for the transferred flap. In the histopathologi- cal examination, no evidence of malignancy was found in the specimen. At 2-month and 1-year follow-up after the last operation, the patient showed a full range of motion with respect to the elbow joint and shoulder with no contracture, as well as healthy skin at the area covered by the flap. There were no complications, such as lymphedema, that sometimes occur during the removal of circumferential nevi (Fig. 4).

\section{DISCUSSION}

Giant congenital melanocytic nevi involve large areas that are more than $20 \mathrm{~cm}$ at the point of the greatest diameter [3], that comprise more than $1 \%$ of the total body surface area in the head and neck region, or that comprise more than $2 \%$ of the total body surface area elsewhere [4]. The face and hands may also display melanocytic lesions [3]. The treatment modalities include non-excisional and excisional methods. The former includes dermabrasion, laser ablation, and chemical peel treatment. The latter include staged or en bloc excision with primary closure, skin substitution or skin grafting, and flap coverage. Non-excisional methods achieve a reduction in the number of superficial nevus cells only, which masks malignant changes of the residual cells in the deep dermis. Therefore, surgical excision is preferable, as it can result in the elimination of all nevus cells. When the lesion is large enough to make conventional flap coverage challenging, clinicians must make use of more sophisticated modalities, like an extended flap, a chimeric flap, or expansion.

If a giant congenital melanocytic nevus affects the entire upper extremity, a local flap may not provide coverage of the full area due to a lack of skin around the defect. Split-thickness skin graft or fullthickness skin graft can provide good coverage of large defects. However, these techniques produce noticeable differences in certain characteristics, such as color and texture, between the healthy skin and the grafted skin. Dysfunction of the anatomical region, 
such as in the joint areas, can occur due to gradual contraction, and these areas require thin, pliable, cushioned coverage due to the potential for ulceration of the grafted skin under continuous mechanical stress [5]. Additionally, skin grafting can lead to morbidity and prolonged healing of the donor site, complicating reconstruction [6]. Skin grafts covering large circumferential defects of the extremities can also cause prolonged swelling or lymphedema of the distal regions, including the hand $[7,8]$. Flap coverage-as in the use of a latissimus dorsi flap with vascularized lymph node transfer or a myocutaneous flap-limits lymphedema of the upper extremities after oncologic surgery $[9,10]$. Therefore, skin grafting is considered to be a secondary measure for coverage of large defects or the joint areas.

Although TE for nevus removal requires multiple stages, this technique is considered a reliable method for the reconstruction of giant nevi to achieve optimum aesthetic and functional outcomes. TE provides extra skin with a more closely matching color and texture and allows repeated use of the expanding donor site.

The TDAP flap is an appropriate choice for several anatomical regions because of its reliable anatomy and ability to be thinned without compromising blood supply, as well as the possibility of harvest with a long pedicle, the pliability of the flap, and the absence of significant donor site morbidity $[11,12]$. Pre-expansion of the TDAP flap can provide extra skin for coverage of the defect with reduced donor site morbidity and can reduce the number of additional operations when the lesion is too large to treat with a single procedure. Moreover, pre-expansion can increase vascularity and improve the safety of the distal regions. Notably, perforator flaps, including TDAP flaps, have the disadvantage of potential damage to perforator arteries. Therefore, the operator should perform careful preoperative evaluation of perforators with Doppler ultrasound and place the tissue expander in a way that avoids direct injury to the vessels. It may also be helpful to include a small amount of muscle and fibrotic tissue around the pedicle.

A disadvantage of TE is that it requires multiple operations. Requiring patients to undergo several procedures may result in added discomfort, increased financial burden, and increased risk of complications, such as infection at the operative site. To overcome these limitations, we planned for just two procedures with a large tissue expander; however, we had to consider the sex of the patient and the patient's typical daily life during the expansion. In our case, we performed subsequent expansion of the TDAP flap primarily at the donor site and secondarily at the recipient site. The patient was pleased with the process and results, despite the financial burden and repeated hospital visits. Use of the unconscious sedative aided in the recovery of the patient from several of the operations. Furthermore, the transferred flap was thin, and provided just enough stability and vascularity for multiple re-expansions [13]. The re-expanded transferred flaps are also sufficient to cover defects from the removal of any newly developed or recurring nevi. Alternative- ly, a free pre-expanded TDAP flap may have also covered the defect, but use of the pedicled flap was preferred considering the arc of rotation of the thoracodorsal arteriovenous pedicle and the ability to make a flap on the ipsilateral side.

A limitation of our process is that it requires several procedures; however, we still achieved minimal scarring and ensured minimal disruption to the patient's daily life. This limitation is due to the use of a relatively small expander at the donor site and the planned reexpansion of the transferred flap, the expansion capability of the skin, subjective symptoms of expansion, and so on. A larger expander, however, can provide a larger amount of skin and reduce the number of operations, but it increases the risk of flap ischemia and skin necrosis, rupture, long scarring, discomfort, and failure of expansion $[14,15]$. Together, the surgeon and the patient can choose a method that meets the goal of the operation through clear discussions.

We present a case of the successful and complete excision of a giant nevus, which nearly spanned the circumference of the upper arm; the resulting defect was covered using a pre-expanded pedicled TDAP flap and staged re-expansion of the transferred flap on the upper arm without complications. Additionally, the use of day surgery in the outpatient department and unconscious sedation were good options for the later procedures that utilized TE.

\section{NOTE}

\section{Conflict of interest}

No potential conflict of interest relevant to this article was reported.

\section{Ethical approval}

The study was approved by the Institutional Review Board of Kyung Hee University Hospital (IRB No. 2019-06-041) and performed according to the principles of the Declaration of Helsinki.

\section{Patient consent}

The patient provided written informed consent for the publication and the use of her images.

\section{ORCID}

Hyee Jae Yang

Sang Yoon Kang

https://orcid.org/0000-0001-8068-8770

https://orcid.org/0000-0002-6299-6371

\section{REFERENCES}

1. Arneja JS, Gosain AK. Giant congenital melanocytic nevi. Plast Reconstr Surg 2009;124(1 Suppl):1e-13e.

2. Corcoran J, Bauer BS. Management of large melanocytic nevi in the extremities. J Craniofac Surg 2005;16:877-85.

3. Kopf AW, Bart RS, Hennessey P. Congenital nevocytic nevi and malignant melanomas. J Am Acad Dermatol 1979;1:123-30. 
4. Lanier VC Jr, Pickrell KL, Georgiade NG. Congenital giant nevi: clinical and pathological considerations. Plast Reconstr Surg1976;58:48-54.

5. Oksuz S, Ulkur E, Tuncer S, et al. Elbow reconstruction with a pedicled thoracodorsal artery perforator flap after excision of an upper-extremity giant hairy nevus. J Plast Reconstr Aesthet Surg 2013;66:566-9.

6. Er E, Ucar C. Reconstruction of axillary contractures with thoracodorsal perforator island flap. Burns 2005;31:726-30.

7. Garza R 3rd, Skoracki R, Hock K, et al. A comprehensive overview on the surgical management of secondary lymphedema of the upper and lower extremities related to prior oncologic therapies. BMC Cancer 2017;17:468.

8. Ortiz CL, Mendoza MM, Sempere LN, et al. Versatility of the pedicled thoracodorsal artery perforator (TDAP) flap in soft tissue reconstruction. Ann Plast Surg 2007;58:315-20.

9. Balakrishnan C, Bradt LM, Khalil AJ, et al. Lymphedema of the upper extremity following circumferential burns. Can J Plast Surg 2004;12: 79-80.
10. Hocaoglu E, Aydin H. Preexpanded perforator flaps of the dorsolateral trunk in pediatric patients. Plast Reconstr Surg 2013;131:1077-86.

11. Wagh MS, Dixit V. Tissue expansion: concepts, techniques and unfavourable results. Indian J Plast Surg 2013;46:333-48.

12. Crosby MA, Dong W, Feng L, et al. Effect of intraoperative saline fill volume on perioperative outcomes in tissue expander breast reconstruction. Plast Reconstr Surg 2011;127:1065-72.

13. Ng ZY, Tan SS, Lellouch AG, et al. Soft tissue reconstruction of complete circumferential defects of the upper extremity. Arch Plast Surg 2017;44:117-23.

14. Inbal A, Teven CM, Chang DW. Latissimus dorsi flap with vascularized lymph node transfer for lymphedema treatment: technique, outcomes, indications and review of literature. J Surg Oncol 2017;115:72-7.

15. Lee KT, Lim SY, Pyun JK, et al. Improvement of upper extremitylymphedema after delayed breast reconstruction with an extended la-tissimus dorsi myocutaneous flap. Arch Plast Surg 2012;39:154-7. 